\title{
Research on the Frequency Spectrum of DC Fault Arc Guo Chen ${ }^{1,2, a}$, Qi Zibo, ${ }^{1,2, b}$ and Gao Wei ${ }^{1,2, c}$
}

\author{
${ }^{1}$ Shenyang Fire Research Institute of Ministry of Public Security, Shenyang, China \\ ${ }^{2}$ Key Laboratory of Fire Scene Investigation and Evidence Identification, Ministry of Public Security, \\ Shenyang, China. \\ aguochen@syfri.cn, bqizibo@syfri.cn, gaowei@syfri.cn
}

Keywords: DC arc; Wavelet; FIR

\begin{abstract}
Since direct fault arc current is lower than the normal current value, it cannot be discovered by over-current protection; On the other hand, the arc is so dangerous. Therefore, it's imperative to research on direct current (DC) arc. In this paper, with a large number of current data through experiments of DC arc, we analyze the data with Haar wavelet transform and FIR (finite impulse response) respectively, and then compare the filtered high frequency signal, proving that Haar wavelet transform can help analyze the frequency spectrum of the arc and recognize the characteristics of DC arc.
\end{abstract}

\section{Introduction}

The electric arc is caused by strong electromagnetic interactions between electrodes, which, for example, are driven apart due to loose contact. In this context, electrodes mean conductor terminals. In the long run, loose contact between electrodes is commonplace, since any wear-out of the terminals, not only cable lines, but also electric components, could result in loose contact, furthermore, arc current. On the other hand, our daily life is rife with direct current, on account of the development of photo-voltaic power generation and electric vehicle. Therefore, the danger of direct current arc fault seems coming.[1]

The existing electric protection protects nothing but overload. However, the arc fault means loss of voltage, which results in the loss of current. This astonishes the existing protection so much that the existing protection can't do nothing.

Even worse, the harm of arc fault is of severity. Arc current of only 0.5 Ampere could result in fire, and of only $2 \mathrm{~A}$ could result in $2000^{\circ} \mathrm{C}$ in some place. Besides, the characteristic of DC arc current, not like AC arc current, is its consistency- there is no process of quenching like AC arc.

Because of the scope, the harm and the difficulty of discovering, DC arc is really a challenge to the electric fire protection, which leads us to analyze the characteristic of arc.

\section{Wavelet Transform of Arc Current}

Fig. 1 is the waveform of the experimental arc current and voltage. The first wave is the voltage of the arc, the second wave is the current, and the third is the induced voltage from the current by mutual inductor. To get such arc current and voltage, we conducted the arc experiment. We made two copper rods with smooth surface closely connected, and then power DC current, which is 6A, on them. When we drove the rods apart, the arc was arisen. The voltage between the two rods is the voltage of the arc, and the current through the rod is the current of the arc. 


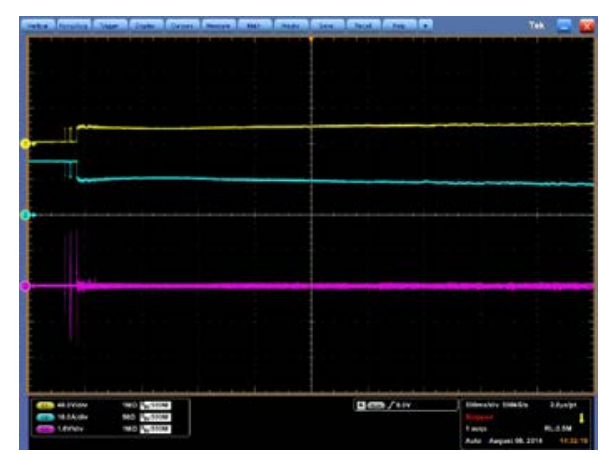

Fig. 1 Waveform of the voltage and current of the arc when initiating the DC arc

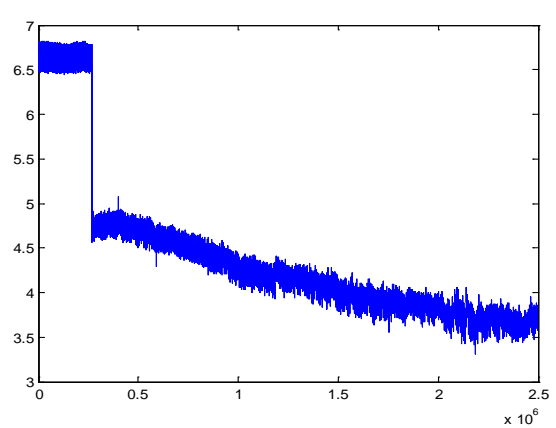

Fig. 1 Current waveform in MATLAB scope

Since the voltage between the rods is impossible to get in real life, we specifically analyze the current of the arc. Before the arc is arisen, the current is produced by the DC voltage, $48 \mathrm{~V}$, loaded on a resistor, $8 \mathrm{Ohm}$. We can see from Fig. 1 that before arc the current is stable, and the voltage of the arc is 0 . When the arc is arisen, the voltage of the arc is emerged, and as a result, the current is decreased. The induced voltage from the current obviously has some peaks, and increased a lot, which means there are varieties of harmonious waves are contained in it.

Due to the lack of period as DC current, the Fourier transform cannot accurately analyze the frequency domain of the DC current. The length of the data can cause a variety of Fourier analysis results, leading to no persuading regular results. Therefore, we choose another kind of method to analyze the characteristics of the arc current, which is wavelet transform.

Fourier analysis consists of breaking up a signal into sine waves of various frequencies. Similarly, wavelet analysis is the breaking up of a signal into shifted and scaled versions of the original (or mother) wavelet.

Compare wavelets with sine waves, which are the basis of Fourier analysis. Sinusoids do not have limited duration -- they extend from minus to plus infinity. And where sinusoids are smooth and predictable, wavelets tend to be irregular and asymmetric.

These two characteristics of this method are perfectly fit to the analysis of DC fault arc current. It's the irregularity and the infinity that really distinguish the arc. I mean, we coincidentally, need to discover the singular point of the current, which is not periodic.

When the sampling data is put into the MATLAB scope, the current waveform is shown as in Fig. 2. The $X$-axis means the number of the sampling point. Since the sampling frequency is $500 \mathrm{kHz}$, the whole time of this waveform is 5 seconds.

In Fig. 2, the sample frequency is $500 \mathrm{kHz}$, and the sample time is 5 seconds. The trend of the current is clearer in Fig.2-the peak and the decrease of the current when the arc happens.

To better analyze the trend, we choose Haar discrete wavelet transform (DWT).

The Haar wavelet's mother wavelet function $\psi(\mathrm{t})$ can be described as shown in Eq. 1.

$$
\Psi(x)=\left\{\begin{array}{c}
1(0 \leq x<1 / 2) \\
-1(1 / 2<=x<1) \\
\text { O(other })
\end{array}\right.
$$

Though this function not practical, but in the analysis of frequency domain, it's much more intuitive than other kind of wavelet due to its similarity with Fourier Transform. For example, Haar wavelet is fit to square wave, and Fourier is fit to sine function. Seemingly not similar, however, a square wave can be described by full frequency domain of sine function. The function relationship is described as Eq. 2.

$$
f(t)=\frac{4 A}{\pi}\left(\cos \omega t-\frac{1}{3} \cos 3 \omega t+\frac{1}{5} \cos 5 \omega t-\frac{1}{7} \cos 7 \omega t+\ldots\right)
$$


We can see from function 2.1 that as the frequency increases, the scalar is much weaker. Therefore, we can believe that with the help of Haar wavelet transform, frequency domain can be analyzed.

According to the analysis of the wave with the help of Haar wavelet, we can get the decomposition of the current waves of varieties of frequencies.

As the sampling data is discrete, one dimensional discrete wavelet transform is chosen to analyze, which can be discomposed to a certain number of levels. For example, when the sampling frequency is $500 \mathrm{kHz}$, the frequency domain of every level is shown as Fig. 3.

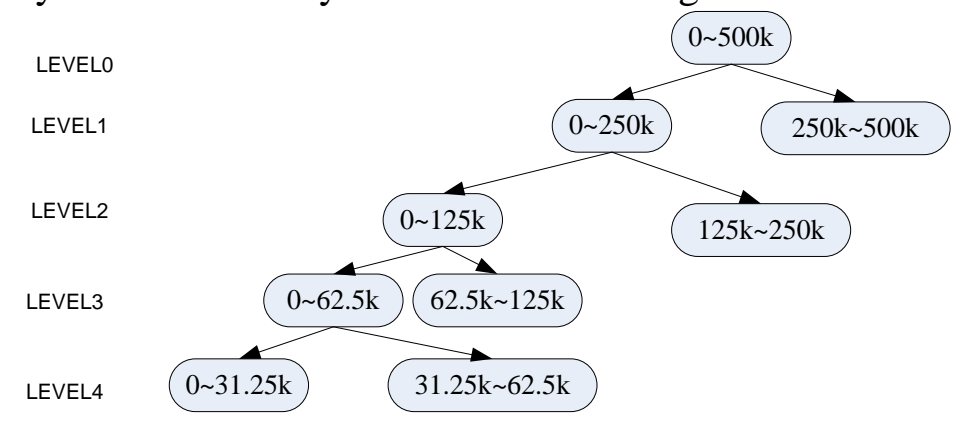

Fig. 2 Frequency domain of every level in wavelet

For many signals, the low-frequency content is the most important part. It is what gives the signal its identity. The high-frequency content, on the other hand, imparts flavor or nuance. In wavelet analysis, we often speak of approximations and details. The approximations are the high-scale, low-frequency components of the signal. The details are high-frequency components.

The MATLAB wavelet toolbox can easily get the the low-scale, high-frequency components.We choose to decompose the waveform to 5 levels, the frequency domain of which is the same as Fig. 3, and the result is as follows.

It can be seen from Fig. 4 that, the decomposition of Level 3 can tell the irregularity of the fault arc current best for the peak is highest in contrast with the effective value in the view of arc current. As level 3 means the frequency domain of the range from $62.5 \mathrm{kHz}$ to $125 \mathrm{kHz}$, we choose this range of frequencies to analyze. The filtered waveform is shown as Fig. 5.

We can see from Fig. 5 that there are lots of peaks contained in the filtered waveform.

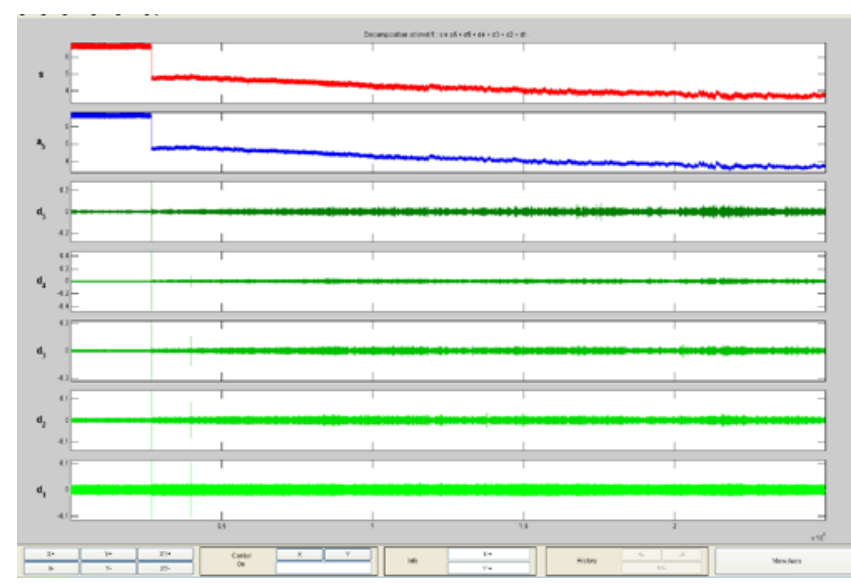

Fig. 3 DWT of the arc current over 5 level

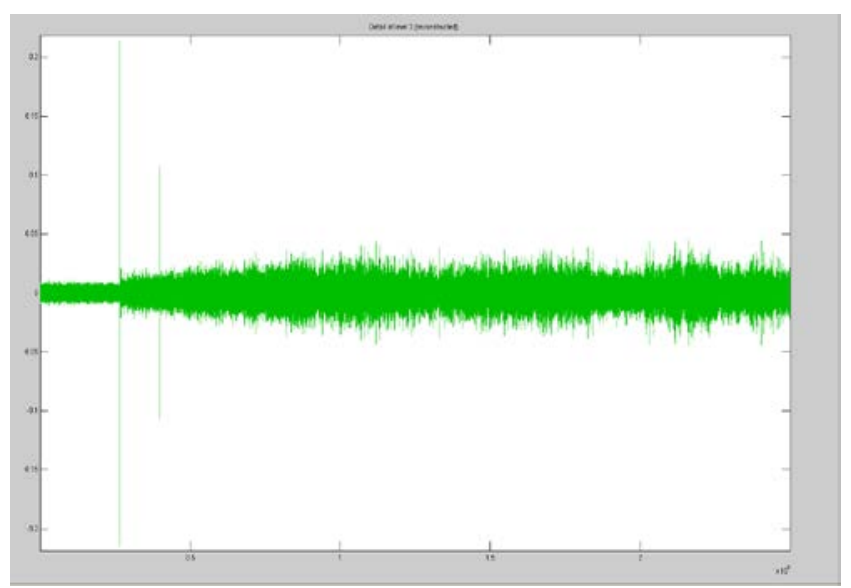

Fig. 4 Waveform of level 3 DWT

\section{Comparision of Filtered Signal Between Dwt And Fir}

References This very range of frequencies can be filtered by means of MATLAB, since MATLAB can simulate the outcome of a specific filter. A digital filter and a wavelet filter are compared to ensure if they are both fit to get the waveform of a specific range of frequency domain.

As for a digital filter, the finite impulse response (FIR) filter is the most stable filter. Therefore, it is also exploited to the analysis of DC arc current, for example, the the waveform of current data as 
shown in Fig. 2. We choose hamming window and 4000 stages to build the filter to get a better view of that band.

Besides, Haar wavelet transform is also used to filter the waveform. The range of frequencies is from $62.5 \mathrm{kHz}$ to $125 \mathrm{kHz}$ as shown in Chapter 2. The outcome of the filter is shown as Fig. 6.

The upper waveform is the outcome of Haar wavelet transform with an offset of 1 , which is to facilitate comparison with the waveform below, which is the output of FIR filter. It can be seen from the Fig. 6 that the trends of the waveforms are almost the same, and the peak is easy to discover.[2]

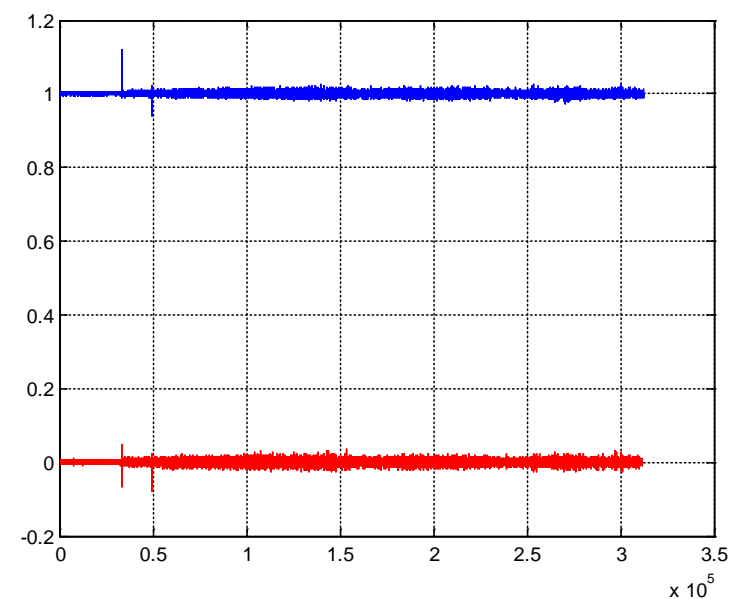

Fig. 6 Comparison of the outcome between DWT and FIR

\section{Summary}

In this paper, the DC fault arc current is sampled and analyzed. Due to the lack of period, Haar wavelet transform is used to analyze the spectral signal in place of discrete Fourier transform. The result of the experiment proves that the wavelet transform is fit for the analysis. Besides, the filtered signal by means of wavelet transform is compared with that of FIR, proving the similarity between these two methods of analyzing arc current, thus proving that the wavelet transform can help analyze the frequency spectrum and lead to the choice of frequency spectrum to filter.

\section{Acknowledgement}

Supported by the Elementary Scientific Project Built for Ministry of Public Security Intensifying the Police by Technology (Grant No. 2013GABJC040), Research on the technology of the detection of DC fault arc. 2013GABJC040

\section{References}

[1] C. Strobl, P. Meckler, Arc Faults in Photovoltaic Systems, submitted to Electrical Contacts Holm Conference(HOLM) .(2010)

[2] J. Johnson, K. Armijo, Parametric study of PV arc-fault generation methods and analysis of conducted DC spectrum , submitted to Photovoltaic Specialist Conference (PVSC). (2014) IEEE 40th ,8-13 June 2014,3543 - 3548 